\title{
Microfocused Ultrasound with Visualization for Face Slimming: Preliminary Results in Four Women
}

\author{
Talitha Possagno Chaves \\ Bellote' \\ Helio Amante Miot (iD ${ }^{2}$ \\ 'Instituto La Beaute (Private Clinic), \\ Curitiba, PR, Brazil; ${ }^{2}$ Departamento de \\ Dermatologia da FMB-Unesp, Botucatu, \\ SP, Brazil
}

Background: Microfocused ultrasound with visualization (MFU-V) is a recently developed technology that improves the laxity of the skin due to its capacity of precise focal heating of the dermis and deep layers, consequently inducing neocollagenesis and elastin remodeling. Purpose: This case series aimed to assess the efficacy of MFU-V in improving skin laxity, leading to the slimming of the lower third of the face in women who desired to naturally improve the shape of their faces.

Patients and Methods: Four women between 29 and 36 years were submitted to a specific protocol with 4-MHz $-4.5-\mathrm{mm}$ and $7-\mathrm{MHz}-3.0-\mathrm{mm}$ transducers (Ulthera ${ }^{\circledR}$ ) at the buccinators region in one session, and patients were evaluated after 180 days. GAIS (Global Aesthetic Improvement Scale) was assessed through standardized photographs (D0 vs D180).

Results: All subjects perceived the slimming of the face after 180 days, as well as the improvement of skin laxity and contour. The practitioner and all the patients were highly satisfied with the results. The assessment of GAIS (D0 vs D180) resulted in score 3 (very improved) for three participants, and score 2 (improved) for one woman. All the patients classified pain experienced during the procedure as mild to moderate.

Conclusion: We reported a technique that uses microfocused ultrasound with visualization leading to the slimming of the lower third of the face. Further systematic studies should be performed to understand the mechanism underneath the results and to maximize its performance.

Keywords: collagen, microfocused ultrasound, neocollagenesis, skin laxity, Ulthera ${ }^{\circledR}$

\section{Introduction}

Facial appearance performs an important social function in the modern world, and modifications of facial shape due to aging and loss of the connective tissue scaffold impairs self-esteem and competitiveness. Thus, the search for improvements in facial harmony is increasing worldwide. One of the standards of beauty sought by women is the triangle of Yarbus, which passes through both eyes with the apex located in the chin. ${ }^{1}$ This triangle however tends to reverse over time due to bone resorption, fat loss, and loss of skin elasticity. ${ }^{2}$

Furthermore, sagging cheeks caused by alterations in subcutaneous adipose layers of the face and loss of skin elasticity are also considered signs of aging faces and may cause concerns, especially in women. ${ }^{3}$

To minimize these effects, many patients search for dermatologists and plastic surgeons to perform aesthetic procedures aimed at normalizing the appearance to
Correspondence: Helio Amante Miot Departamento de Dermatologia da FMB-Unesp, Rua Magnólia, 400, Jd. Bom Pastor, Botucatu, SP, 18.607-670, Brazil Tel +55 1438134727

Email heliomiot@gmail.com 


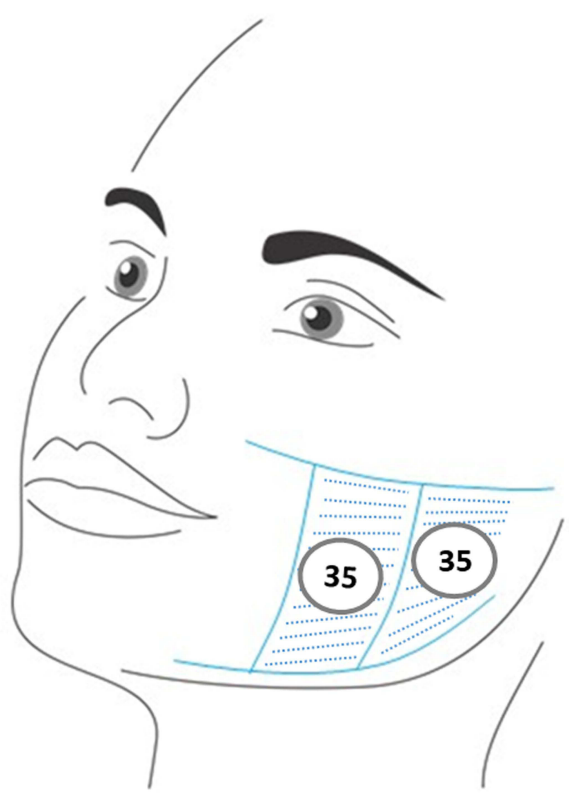

4-MHz, 4.5-mm transducer

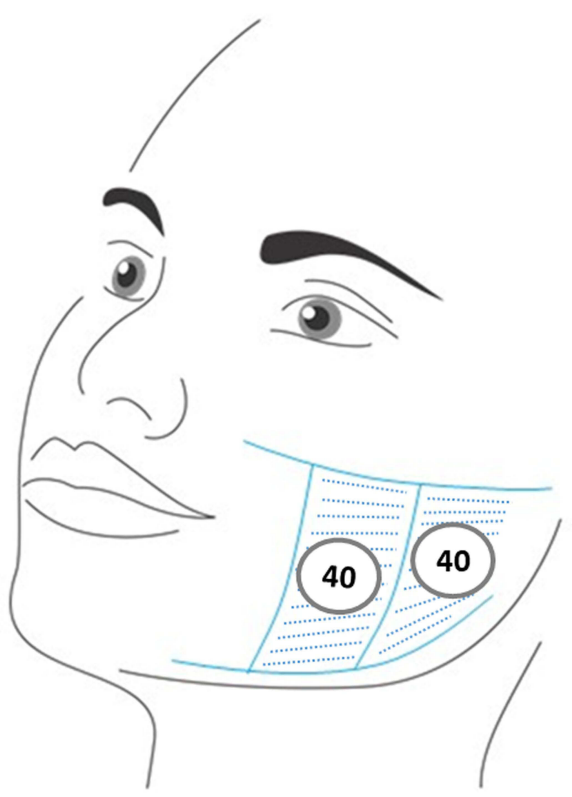

7-MHz, 3.0-mm transducer

Figure I First area delimited by two vertical zones: anterior and posterior. Note the distance of $2.5 \mathrm{~cm}$ between the anterior line and oral commissure (security area). The inclination of the transducer was oriented to perform the contraction in direction to the tragus.

the Yarbus triangle and other facial treatments. Surgical procedures, such as buccal fat pad removal (bichectomy), have been indicated, despite higher risks than noninvasive procedures. $^{4}$

Microfocused ultrasound with visualization (MFU-V) has been used as a treatment for skin laxity with good results ${ }^{5}$ due to its capacity to propagate through tissue and cause small microthermal zones of coagulation in the deep dermis and the subjacent connective tissue. The thermal damage process initiates a wound-healing response that results in the production of new collagen and elastic fibers, leading to tissue contraction, improvement of skin elasticity, and dermal thickening. In addition, visualization allows treatment to target the desired tissue, customizing it according to the rationale of the expected result in each layer with increased safety. ${ }^{6-8}$

MFU-V provides a sculpting effect if the subcutaneous tissue is targeted due to thickening of the fibrous septae in the fat layer and SMAS; however, the method does not consume fat and is not considered a fat-treatment technology. ${ }^{8}$

Actually, MFU-V has become a reproducible treatment for facial subdermal lifting and skin tightening, which results varies according to the site of application, focal depth, energy, vectoring lines, and serial treatment plans. ${ }^{7-}$ ${ }^{9}$ The combination of these features allows to the development of different protocols to be further validated. In this case series, we report a novel protocol of MFU-V treatment with Ulthera ${ }^{\circledR}$ which result in the slimming of the lower third face.

\section{Patients and Methods}

Four patients with mild skin laxity and the desire to tighten the face were submitted to the following protocol. Thirty minutes before the procedure, a topical anesthetic with $23 \%$ lidocaine and $7 \%$ tetracaine formulation was applied, and $750 \mathrm{mg}$ of acetaminophen was orally administered. Ten minutes before the start of the procedure, $10 \mathrm{mg}$ of trometamol was orally administered to minimize pain during the session.

While waiting for the anesthetic effects to occur, the treatment area was divided in two regions. The first treatment area was limited by an anterior line $2.5 \mathrm{~cm}$ apart from oral commissures, a posterior line anterior to tragus, a superior line below the maxilla and zygomatic arch and an inferior line above the jaw line (Figure 1).

The second treatment area lies within the first treatment, and it is limited by the same anterior and posterior lines of the first area. However, the superior line is localized approximately $1 \mathrm{~cm}$ below the zygomatic arch, and the inferior line is located $1 \mathrm{~cm}$ above the jaw line (Figure 2), to intensify the contraction of the underneath tissue in the direction of the tragus. Both areas were divided in two columns (anterior and posterior) to properly receive the transducers. 


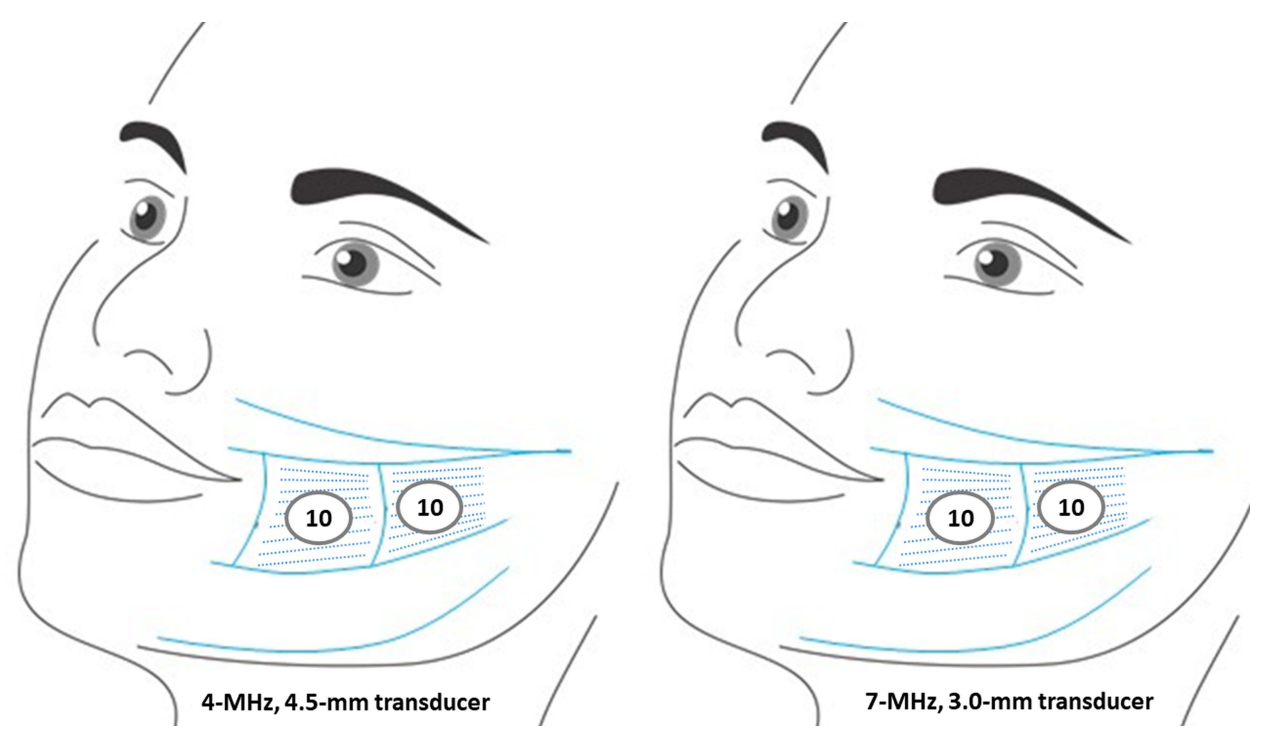

Figure 2 Second area delimited, the treatment is concentrated within two zones: anterior and posterior, which are narrower than the first area. The inclination of the transducer was oriented to perform the contraction in direction to the tragus.

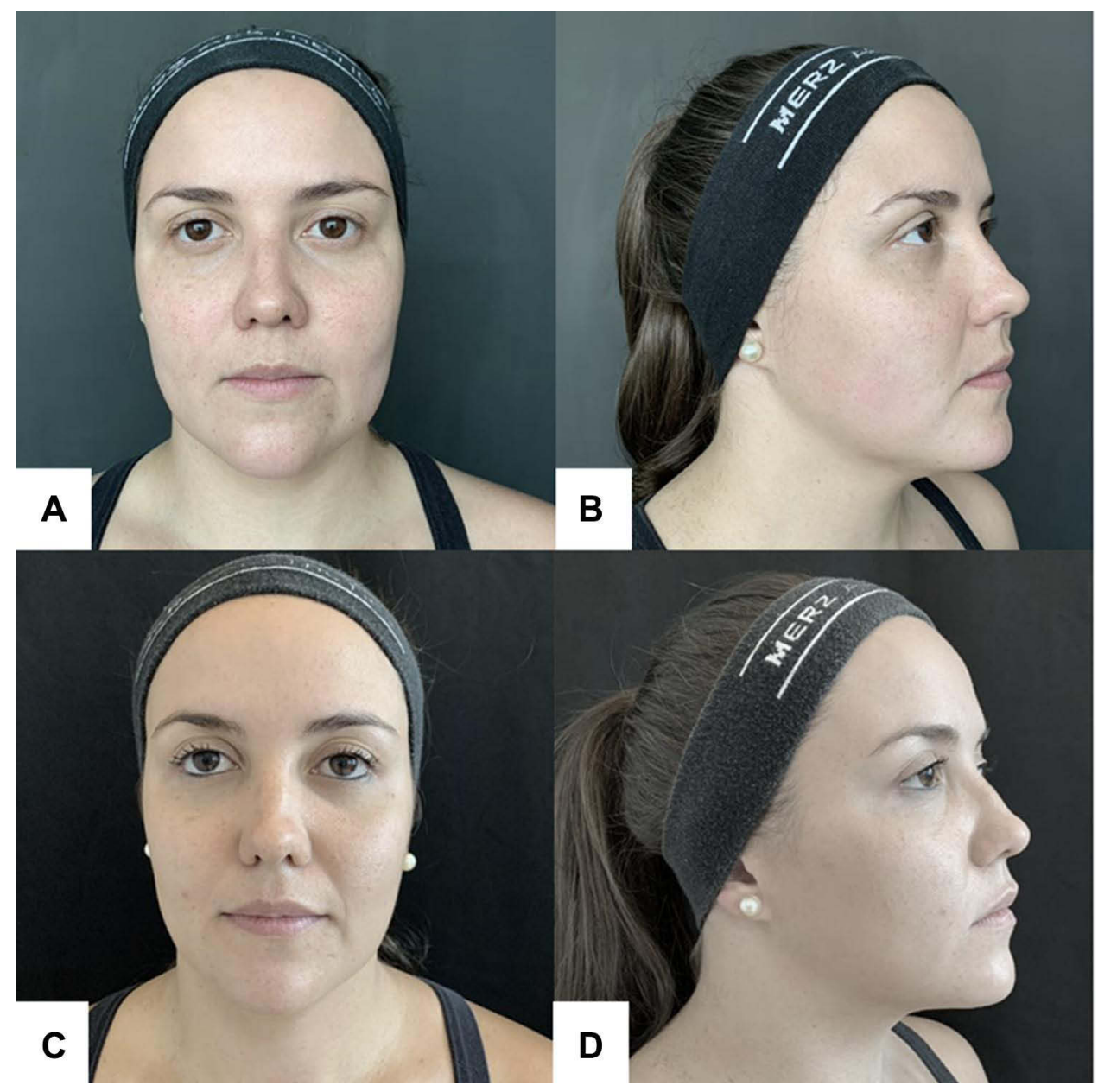

Figure 3 Woman, 33 years old; (A and B) Before procedure; (C and D) 180 days after procedure: slimming of the middle third of the face and improvement of the jawline. 


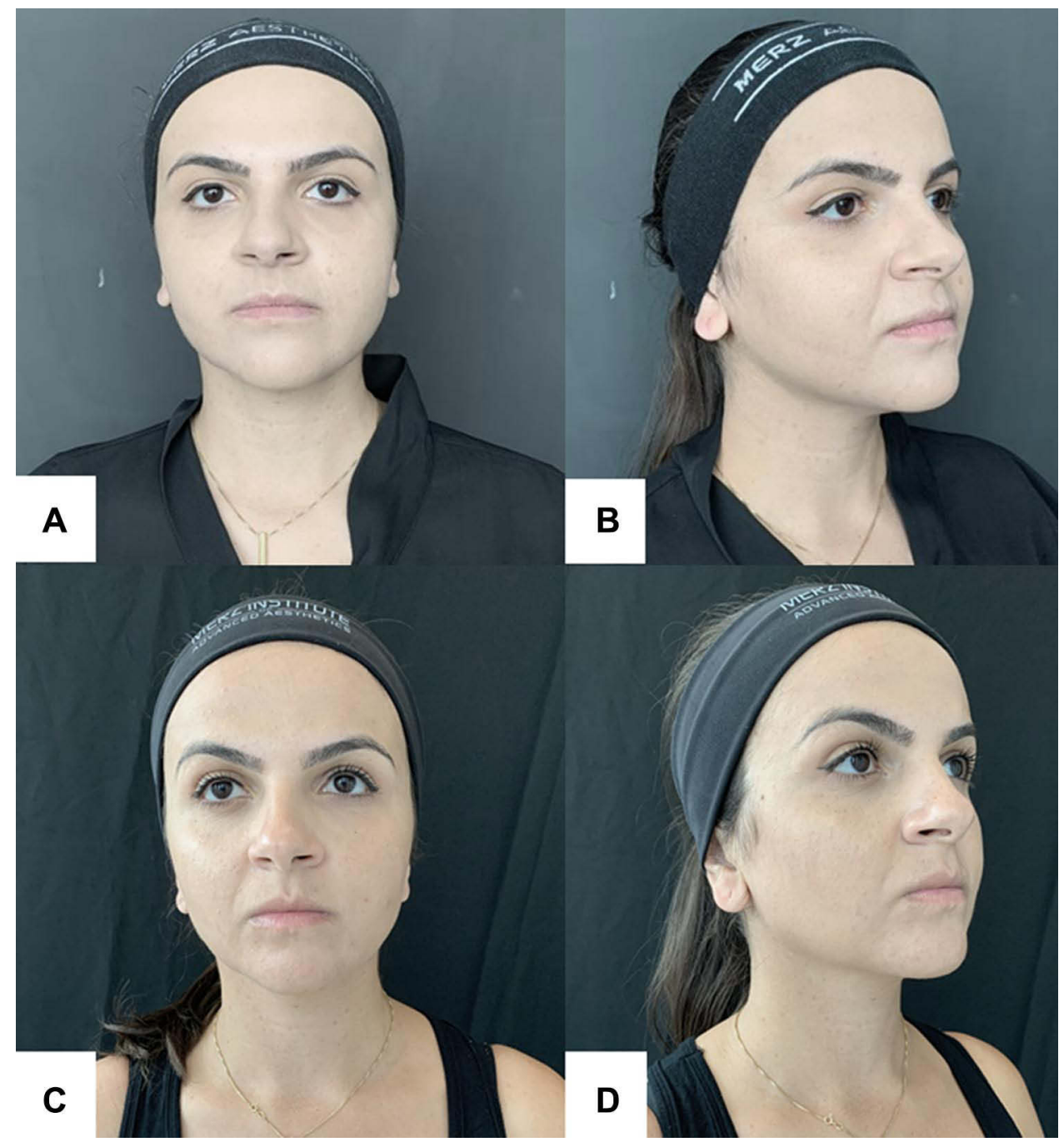

Figure 4 Woman, 36 years old; (A and B) Before procedure; (C and D) 180 days after procedure: discrete slimming of the middle third of the face and marked improvement of jawline 180 days after procedure.

After delimitation, the topical anesthetic was washed off, and an ultrasound gel was applied to the skin immediately before energy delivery. The technology used in all the cases was Ulthera ${ }^{\circledR}$ (Merz Aesthetics ${ }^{\circledR}$, Frankfurt, Germany) based on the following protocol. First, 4-MHz $-4.5-\mathrm{mm}$ transducers were applied in the first area with 35 homogeneous shots per vertical column and, in area two, with 10 concentered shots each column.

Afterwards, a 7-MHz-3.0-mm transducer was applied in area one with 40 homogeneous shots for each vertical column and 10 concentered shots for each column in the second area.

The transducers were reoriented at each shot advancing $1 \mathrm{~mm}$ up, in both areas, to perform the contraction in the direction to the tragus. The treatment areas in this protocol are narrower than the area used in the standardized protocols for Ulthera ${ }^{\circledR}$. And these restricted areas were chosen aiming to create a reduction in the volume and a pattern of contraction leading to the appearance of slimming on the lower third of the face.

Previously to the treatment, the area was scanned and SMAS was visualized in all the patients, to guarantee the SMAS was reached by the transducer $4-\mathrm{MHz}-4.5-\mathrm{mm}$ in all cases, as well as the transducer $7-\mathrm{MHz}-3.0-\mathrm{mm}$ reached the subcutis.

Patients were analyzed by standardized photographs at D0 and D180 and asked about improvement and satisfaction at D90 and D180. The Global Aesthetic Improvement Scale (GAIS) was used to assess the difference in the global appearance of the face through standardized photographs (D0 vs D180). Two blinded evaluators (T.P.C.B. and H.A.M.) consensually assessed the photographs using 


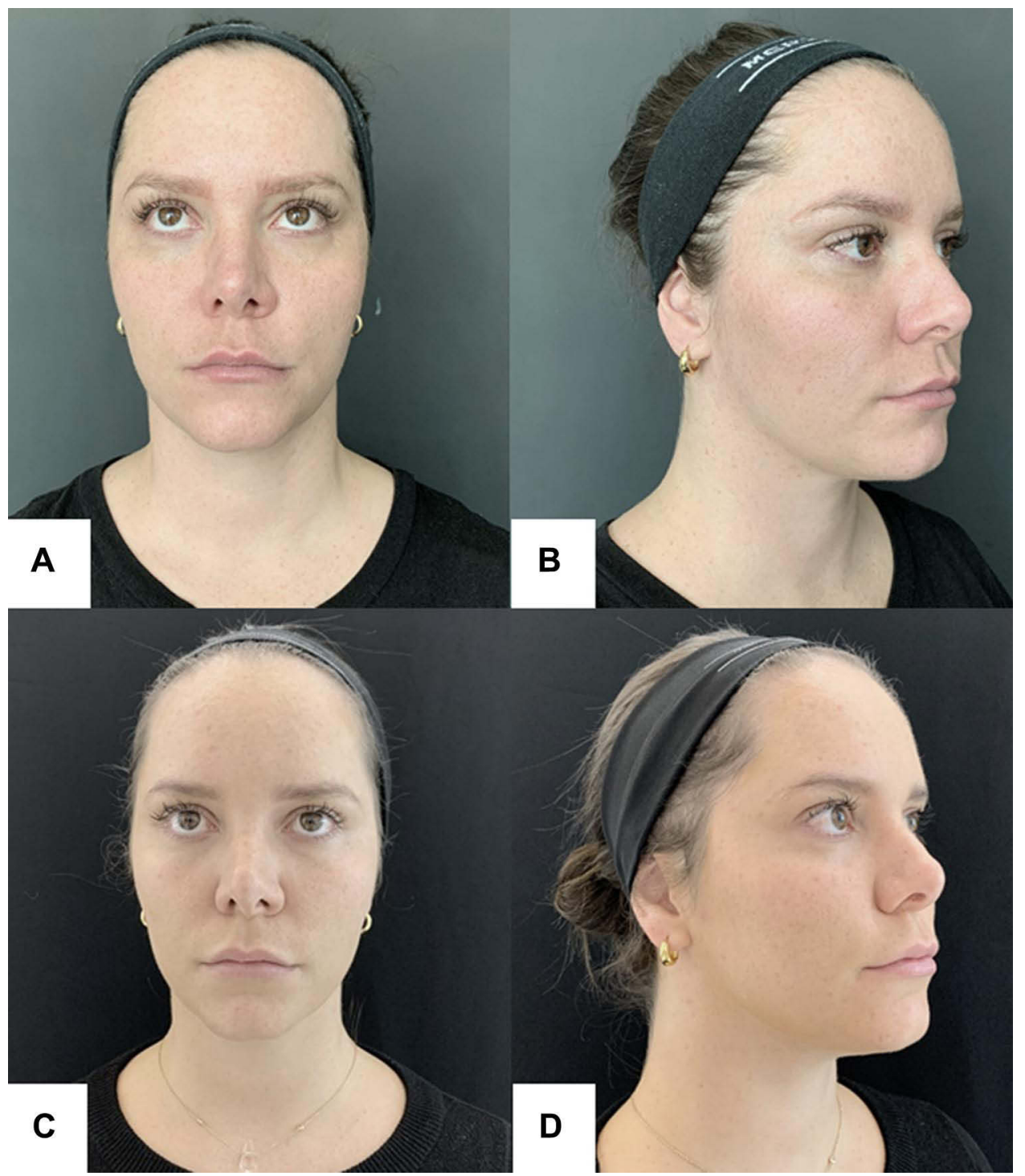

Figure 5 Woman, 29 years old; (A and B) Before procedure; (C and D) 180 days after procedure: evident slimming of the middle third of the face, turning more evident the triangle format of the face (Yarbus triangle).

a 5-point ordinal scale $(4=$ exceptionally improved patient, 3 = very improved patient, 2 = improved patient, $1=$ unaltered patient, $0=$ worsened patient).

Ethics committee or institutional review board approval was not necessary for the individual cases reported in this series because each case reflects a retrospective description of clinical findings, moreover all the subjects gave the written consent for data and image publication.

\section{Results}

The images of the four participants at D0 and D180 are presented in Figures 3-6. The practitioner (T.P.C.B.) was satisfied with the results, and concerning the patients' appreciation, all them were highly satisfied with the results and they are willing to perform the procedure again.

The assessment of GAIS (D0 vs D180) resulted in score 3 (very improved) for three participants, and score 2 (improved) for one woman.

During the procedure, patients experienced mild to moderate pain in the treated areas. No adverse events or pharmacologic toxicity were reported after the procedure in any of the visits.

\section{Discussion}

The aging face is inflicted by changes in skin texture, volume, shape, elasticity, and consistency. ${ }^{9}$ Currently, 


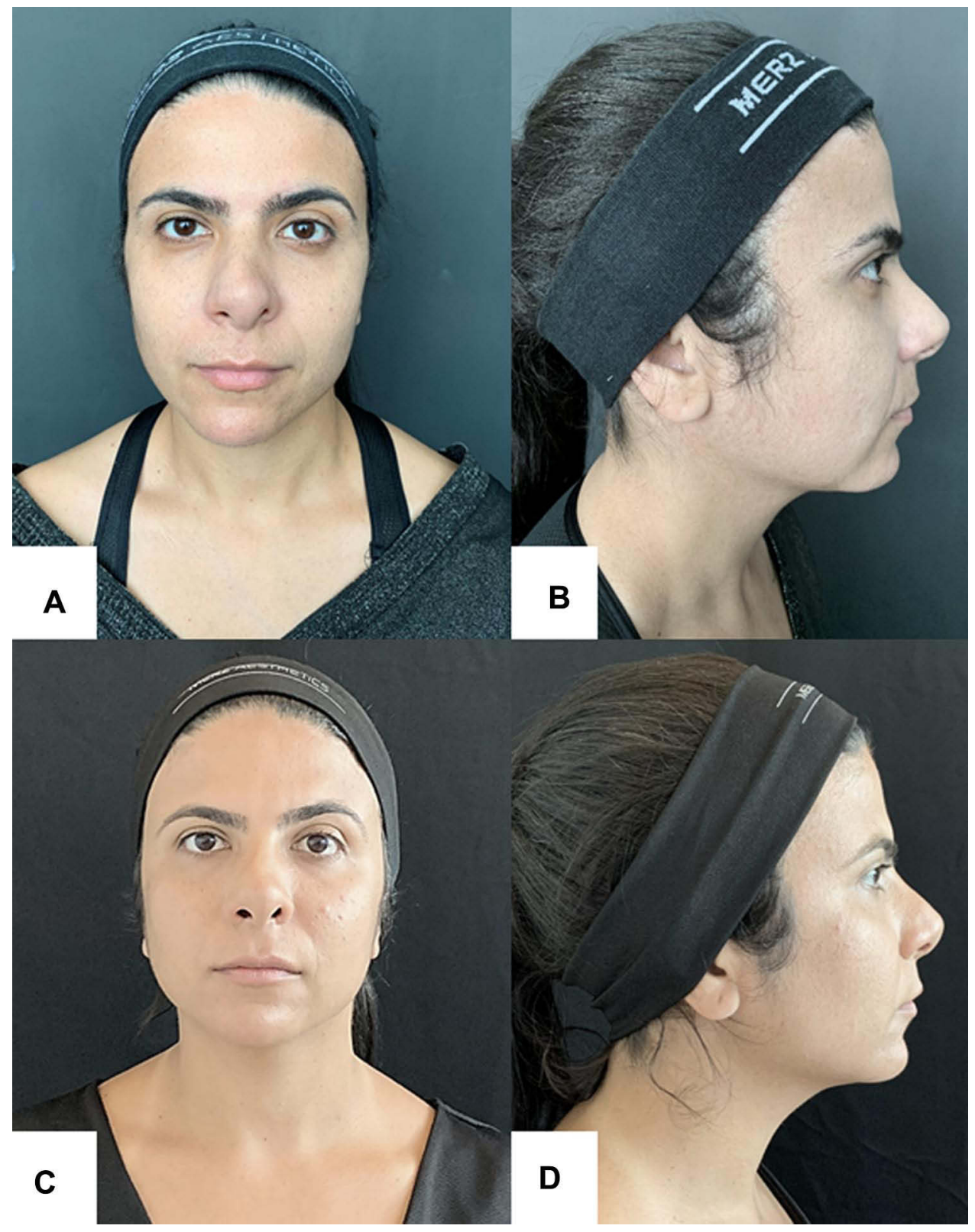

Figure 6 Woman, 34 years old; (A and B) Before procedure; (C and D) 180 days after procedure: slimming of the middle third of the face and improvement of the skin laxity.

with advancing life expectancy, the search for rejuvenation procedures is increasing worldwide. While excisional face lifting surgery is well established and has marked efficacy, patients are concerned about visible scars, risk of postprocedure complications and recovery time. The middle third of the face is an area that enlarges with age; thus, the appearance of the Yarbus triangle is lost. Therefore, new minimally invasive strategies, such as MFU-V, to improve the middle third of the face are being increasingly used in clinics. Additionally, the visual effect of a slim face in its middle third improves the appearance due to sustained Yarbus triangle appearance. ${ }^{1,3}$

Noninvasive procedures, such as MFU-V, are the preferred treatments of the middle third of the aging face. In our cases, despite the lack of a control group or another procedure for comparison, all our subjects indicated improvement in the area. In addition, the photographs also registered a substantial improvement in the shape of the face.

Several previous studies with MFU-V were performed and focused on evaluating skin laxity, and improving the aging 
face. To date, no study has evaluated MFU-V therapy aiming to slim the face, as assessed in this case series. Concerning tightening and facial rejuvenation, studies have reported improvements in skin laxity and skin quality. ${ }^{10}$

The mechanism by which MFU-V leads to face slimming should be investigated through well-designed protocols. As long MFU-V does not promote fat destruction, the tightening of the fibrous septae at the fat layer and the SMAS, promoting a tridimensional contraction, is the best hypothesis for the results. ${ }^{8}$ In addition, the treatment of marionette folds with MFU-V promotes a similar kind of contraction, which can be modulated according to the deep and the intensity of the MFU-V treatments. ${ }^{11}$

It is important to emphasize that the quality of outcomes depends on the correct indication and application technique to maximize the results and to achieve major patient satisfaction. ${ }^{7}$ MFU-V may be combined with other treatments, such as dermal fillers, biostimulators, toxins and other devices, such as radiofrequency, with good results regarding skin laxity reported in the literature. ${ }^{10,11}$

In this preliminary results in four women, objective measures (eg, superimposed standardized pictures, volume calculation, validated scales, and the thickness of the dermis) were not used to assess the results, in addition, the small number of participants would lead to an underpowered statistical analysis. Nevertheless, the visible face slimming provided by this technique may stimulate the scientific community to perform clinical trials assessing MFU-V as a treatment to slim the face. Further systematic prospective study is warranted.

\section{Conclusion}

We reported a technique that uses microfocused ultrasound with visualization leading to the slimming of the lower third of the face. Further systematic studies should be performed to understand the mechanism underneath the results, and to maximize its performance.

\section{Informed-Consent Statement}

Written informed consent was obtained from patients to publish this paper.

Clinical, Cosmetic and Investigational Dermatology

Publish your work in this journal

Clinical, Cosmetic and Investigational Dermatology is an international, peer-reviewed, open access, online journal that focuses on the latest clinical and experimental research in all aspects of skin disease and cosmetic interventions. This journal is indexed on CAS.

\section{Acknowledgments}

To the patients who allowed to publish their photos. Merz has provided the transducers used in this report.

\section{Disclosure}

Hélio Amante Miot is a medical consultant of Merz, and L 'Oreal. Talitha Possagno Chaves Bellote is a speaker from Merz. The authors report no other conflicts of interest in this work.

\section{References}

1. Springer IN, Zernial O, Wiltfang J, et al. Facial aesthetics part I - the significance of the triangle of Yarbus. Mund Kiefer Gesichtschir. 2007;11(3):145-151. doi:10.1007/s10006-007-0057-5

2. Mendelson $\mathrm{B}$, Wong $\mathrm{CH}$. Changes in the facial skeleton with aging: implications and clinical applications in facial rejuvenation. Aesthetic Plast Surg. 2020;44(4):1151-1158. doi:10.1007/s00266-020-01823-x

3. Hennekam RCM. The external phenotype of aging. Eur J Med Genet. 2020;63(11):103995. doi:10.1016/j.ejmg.2020.103995

4. Moura LB, Spin JR, Spin-Neto R, et al. Buccal fat pad removal to improve facial aesthetics: an established technique? Med Oral Patol Oral Cir Bucal. 2018;23(4):e478-e484. doi:10.4317/medoral.22449

5. Fabi SG, Massaki A, Eimpunth S, et al. Evaluation of microfocused ultrasound with visualization for lifting, tightening, and wrinkle reduction of the décolletage. $J$ Am Acad Dermatol. 2013;69(6):965971. doi:10.1016/j.jaad.2013.06.045

6. Suh DH, So BJ, Lee SJ, et al. Intense focused ultrasound for facial tightening: histologic changes in 11 patients. $J$ Cosmet Laser Ther. 2015;17(4):200-203. doi:10.3109/14764172.2015.1007065

7. Fabi SG, Joseph J, Sevi J, et al. Optimizing patient outcomes by customizing treatment with microfocused ultrasound with visualization: gold standard consensus guidelines from an expert panel. $J$ Drugs Dermatol. 2019;18(5):426-432.

8. Friedman O, Isman G, Koren A, et al. Intense focused ultrasound for neck and lower face skin tightening a prospective study. $J$ Cosmet Dermatol. 2020;19(4):850-854. doi:10.1111/jocd.13313

9. Brobst RW, Ferguson M, Perkins SW. Ulthera: initial and six month results. Facial Plast Surg Clin North Am. 2012;20(2):163-176. PMID: 22537784. doi:10.1016/j.fsc.2012.02.003

10. Kwon HH, Lee WY, Choi SC, Jung JY, Bae Y, Park GH. Combined treatment for skin laxity of the aging face with monopolar radiofrequency and intense focused ultrasound in Korean subjects. $J$ Cosmet Laser Ther. 2018;20(7-8):449-453. doi:10.1080/ 14764172.2018.1427870

11. Sasaki GH, Abelev N, Papadopoulos L. A split face study to determine the significance of adding increased energy and treatment levels at the marionette folds. Aesthet Surg J. 2017;37(8):947-960. doi:10.1093/asj/sjx055
The manuscript management system is completely online and includes a very quick and fair peer-review system, which is all easy to use. Visit http://www.dovepress.com/testimonials.php to read real quotes from published authors. 